Xiaoming Lou*, Mingwu Sun and Jin Yu

\title{
Stress wave propagation in different number of fissured rock mass based on nonlinear analysis
}

https://doi.org/10.1515/ijnsns-2019-0301

Received December 14, 2019; accepted October 17, 2020; published online November 23, 2020

\begin{abstract}
The fissures are ubiquitous in deep rock masses, and they are prone to instability and failure under dynamic loads. In order to study the propagation attenuation of dynamic stress waves in rock mass with different number of fractures under confining pressure, nonlinear theoretical analysis, laboratory model test and numerical simulation are used respectively. The theoretical derivation is based on displacement discontinuity method and nonlinear fissure mechanics model named BB model. Using ABAQUS software to establish a numerical model to verify theoretical accuracy, and laboratory model tests were carried out too. The research shows that the stress attenuation coefficient decreases with the increase of the number of fissures. The numerical simulation results and experimental results are basically consistent with the theoretical values, which verifies the rationality of the propagation equation.
\end{abstract}

Keywords: confining pressure; fissured rock; nonlinear analysis; stress wave.

\section{Introduction}

Deep underground engineering construction is the important contents for research in the field of modern civil engineering. Compared with the shallow part, the deep rock mass has the characteristics of high confining pressure, high temperature, high osmotic pressure and strong time effect. Which makes the organization structure, basic behavioral characteristics and engineering response of the deep rock mass change fundamentally compared to the shallow part. Deep rock masses exhibit obvious nonlinear characteristics, forcing deep underground engineering to establish entirely new mechanical systems, theoretical and technical methods. The fissures are ubiquitous in deep rock masses, and studying the propagation of stress waves in cracked rock masses under high confining pressure will have important guiding significance for deep underground engineering excavation.

There are large number of fissures in the wall rocks of the deep underground engineering area, and the existence of these cracks has greatly changed the propagation law of stress waves. Plenty of researches had been carried out by industry scholars on the attenuation law of stress waves in cracked rock mass. The theoretical studies are divided into two categories: one is the displacement discontinuity method (DDM) proposed by Schoenberg [1], this theory argues that when the stress wave passes through the fissures, the stress remains continuous, but the displacement is discontinuous; the other category is based on the extension of DDM, including the method of characteristics [2], the scattering matrix method [3], and the virtual wave source [4], etc. In addition, Bandis et al. [5] proposed a hyperbolic BB model describing the phase nonlinear deformation characteristics of the fissure method, and it is widely used in researches of various cracks, which has

*Corresponding author: Xiaoming Lou, College of Zijin Mining, Fuzhou University, Fuzhou, Fujian, 350116, China; and Institute of Blasting Technology, Fuzhou University, Fuzhou, 350116, China, E-mail: 331261323@qq.com. https://orcid.org/0000-0002-91154368

Mingwu Sun, College of Zijin Mining, Fuzhou University, Fuzhou, Fujian, 350116, China; and Institute of Blasting Technology, Fuzhou University, Fuzhou, 350116, China

Jin Yu, Institute of Geotechnical Engineering, Huaqiao University, Xiamen, 361021, China

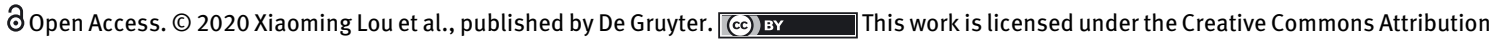
4.0 International License. 
promoted the theoretical development of stress wave penetrating fissures. In terms of experimental research, $\mathrm{Li}$ and Ma [6] pointed out by SHPB test that the existence of fissures will influence the propagation of stress waves, and the stress attenuation coefficient is closely related to the fissures in rock mass; Tingting et al. [7] studied the attenuation law of stress waves passing through a group of parallel fissures, and concluded the relationship between the attenuation coefficient and the number of fissures. Pyrak-Nolte [8] studied the main factors causing the stress wave attenuation; $\mathrm{Yu}$ [9] studied the attenuation characteristics of the stress waves that are incident perpendicularly to the fissured rock mass; Liu Tingting et al. [15] and Chai Shaobo et al. [16] studied the propagation of plane stress wave in nonlinear jointed rock mass using UDEC system, and concluded that the joint spacing and joint quantity jointly affect the wave propagation characteristics.Chai Shaobo et al. [17] studied the propagation characteristics of plane $\mathrm{P}$ wave in nonlinear cross joints through theory and UDEC method, and concluded that joint stiffness, distribution and incident wave frequency all affect the wave propagation process. Li Xinping et al. [18] studied the propagation and attenuation law of cylinder wave in intact rock and jointed rock mass under different in-situ stresses respectively through theoretical analysis and model test; Tingting Liu et al. [19] studied the influence of ground stress on wave propagation through Barton-Bandis (B-B) and hyperbolic nonlinear (HN) slip model, which is not only related to gravity and tectonic stress, but also related to the direction of particle vibration of incident wave.

The existing research results mainly focus on the propagation of stress waves in shallow fissured rock masses, and there are few studies on the propagation law of stress waves in different number of fissured rock mass under the influence of confining pressure. Although industry scholars have studied the influence of fissures on the propagation of stress, they have not combined with the confining pressure factor to discuss it indepth. Through theoretical analysis, laboratory model test and numerical simulation, this paper focusses on the influence of the number of fissures on the stress wave propagation in rock mass under different confining pressures, and compares the derived theoretical results of the attenuation equation with the experimental results and numerical simulation results for verification, which has provided a new idea for the study of the propagation and attenuation of stress wave in fissured rock mass under different confining pressures.

\section{Theoretical basis}

\subsection{Nonlinear fissure mechanics model}

In the nonlinear fissure mechanics model named BB model proposed by Bandis et al. [5], the relationship between the stress acting on the fissure and the amount of fissure closure is determined as:

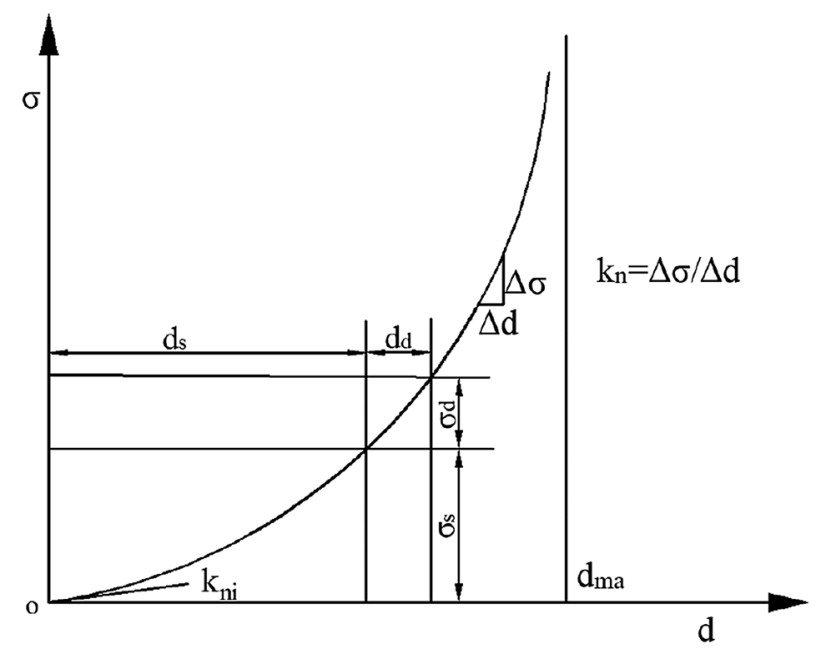

Figure 1: Deformation characteristics of cracks under initial stress. 


$$
d=\frac{\sigma}{k_{\mathrm{ni}}+\sigma / d_{\mathrm{ma}}}
$$

where $k_{\mathrm{ni}}$ is the initial normal stiffness of the crack, $d_{\mathrm{ma}}$ is the maximum amount of crack closure. Under the action of the initial confining pressure $\sigma_{s}$, the relationship between the stress received by the crack $\sigma$ and the amount of crack closure $d$ is shown in Figure 1.

It can be seen from Figure 1 that when the stress wave propagates to the crack with the initial confining pressure, the closing amount of the crack is composed of the static closing amount $d_{s}$ generated by the initial confining pressure and the dynamic closing amount $d_{d}$ generated by the stress wave.

\subsection{Propagation equation of stress wave in rock mass with multiple fissures}

\subsubsection{Mechanical model of multiple fissured rock mass under confining pressure}

A mechanical model with confining pressure $\sigma_{s}$, stress wave P-wave, and $n$ fissures was drawn as shown in Figure 2. Assume the rock material on both sides of the fissures are linear elastic materials with isotropic properties, the incident P-wave produces a reflected P-wave on the left side of the first fissure, and a transmitted P-wave on the right side of the $n$th fissure. The confining pressure $\sigma_{s}$ is isotropic in the rock mass, and the stress $\mathrm{P}$-wave undergoes multiple transmission and reflection between the fissures.

\subsubsection{Stress propagation equations of multiple fissures under confining pressure}

Based on DDM [1] and BB model [5], the propagation focus on the condition that the stress wave propagation direction is consistent with the crack normal direction. According to the law of wave front momentum conservation and the interaction between stress wave and fissures [10], we can conclude that when the P-wave is perpendicularly incident on multiple parallel fissures, the left and right dynamic stresses $\sigma_{d}^{-}$and $\sigma_{d}^{+}$of the $m$ th fissure are:

$$
\begin{aligned}
& \sigma_{d}^{-}=z\left(v_{r}^{-}-v_{l}^{-}\right) \\
& \sigma_{d}^{+}=z\left(v_{r}^{+}-v_{l}^{+}\right)
\end{aligned}
$$

The vibration velocities $v_{p}^{-}$and $v_{p}^{+}$of the particles on the two sides of the fissure are:

$$
\begin{aligned}
& v_{p}^{-}=v_{r}^{-}-v_{l}^{-} \\
& v_{p}^{+}=v_{r}^{+}-v_{l}^{+}
\end{aligned}
$$

where the superscripts “-” and “+” indicate the left and right sides of the fissure, respectively. $v_{r}^{-}$and $v_{l}^{-}$represent the vibration velocities of left-going and right-going P-waves on the left side of the fissure. $v_{r}^{+}$and $v_{l}^{+}$represent the vibration velocities of left-going and right-going P-waves on the right side of the fissure. $z$ is the wave impedance of the rock, $z=\rho c$, where $c$ is the propagation velocity of the stress wave, and $\rho$ is the density of the rock.

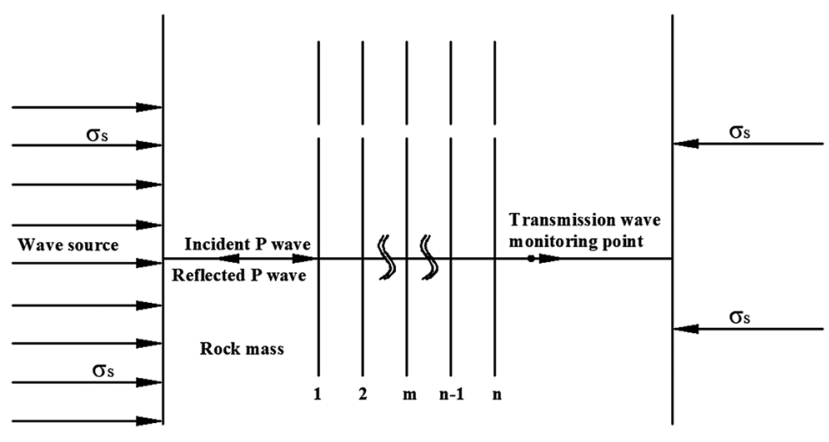

Figure 2: Stress propagation model of multiple fissures under confining pressure. 
According to the (DDM), stress on both sides of the fissure is continuous and the displacement on both sides of the fissure is discontinuous, the stress-displacement relationship on both sides of the fissure is obtained as:

$$
\begin{aligned}
& \sigma=\sigma_{s}+\sigma_{d}^{-}=\sigma_{s}+\sigma_{d}^{+} \\
& u=d=u_{d}^{-}-u_{d}^{+}
\end{aligned}
$$

Take the derivative of Eq. (7) with respect to time, we can get:

$$
\begin{aligned}
v_{p}^{-}(i)-v_{p}^{+}(i) & =\left[\sigma_{d}^{+}(i+1)-\sigma_{d}^{-}(i+1)\right] / k \Delta t \\
k & =\left[k_{\mathrm{ni}}+\left(\sigma_{s}+\sigma_{d}^{+}(i)\right) / d_{\mathrm{ma}}\right]^{2} / k_{\mathrm{ni}}
\end{aligned}
$$

where: $v_{p}^{-}(i)$ and $v_{p}^{+}(i)$ are the vibration velocities of the particle on both sides of the fissure at time $i, \Delta t$ is the time step.

$$
\begin{aligned}
v_{l}^{-}(i) & =v_{r}^{+}(i)+v_{l}^{+}(i)-v_{r}^{-}(i) \\
v_{r}^{+}(i+1) & =(1-A) v_{r}^{+}(i)+(1+A) v_{l}^{+}(i)+A v_{r}^{-}(i)-A v_{l}^{-}(i)-v_{l}^{+}(i+1)
\end{aligned}
$$

where $A$ is the peak stress after passing through the cracks.

When the stress wave P-wave propagates through multiple fissures, assume the rock materials between the fissures are linear elastic materials, then the P-wave satisfies the time-shifting function [13]:

$$
\begin{aligned}
& v_{r}^{-}(i)_{m}=v_{r}^{+}\left(i-n_{p}\right)_{\mathrm{m}-1} \\
& v_{l}^{+}(i)_{m}=v_{l}^{-}\left(i-n_{p}\right)_{\mathrm{m}+1}
\end{aligned}
$$

Similarly:

$$
v_{l}^{+}(i+1)_{m}=v_{l}^{-}\left(i+1-n_{p}\right)_{\mathrm{m}+1}
$$

where nis the number of time-shifting of P-wave between two adjacent fissures, and the formula is as follows:

$$
n=\operatorname{int}[S /(c \Delta t)]
$$

In the formula, $S$ is the fissure spacing, constitute simultaneous Eqs. (3)-(18), and substitute the initial conditions and boundary conditions of the mechanical model, then we can get the fissure attenuation coefficient $T$ :

$$
T=\max \left|v_{r}^{+}(i)_{n} / v_{r}^{-}(i)_{1}\right|
$$

According to Eq. (16), write Mathematica programs to calculate the attenuation coefficient of stress wave when it passing through a certain number of fissures under different confining pressures.

\section{Model test}

\subsection{Test system and test program}

\subsubsection{Stress test principle and test system}

At present, strain gauge is a main way to measure the blasting stress, and it has the merits of repeatable monitoring, low cost and high precision [11]. The model test adopted the strain gauge of model 120-50AA with a resistance value of $120 \Omega$, a grid size of $50.0 \mathrm{~mm} \times 4.0 \mathrm{~mm}$, and a sensitivity coefficient of $2.08 \pm 1 \%$. The test 


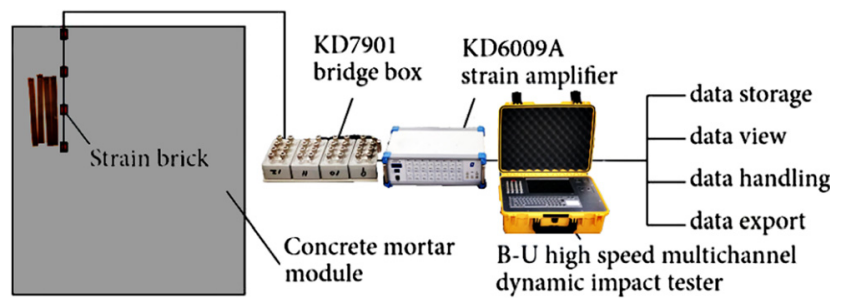

Figure 3: Test system for stress monitoring.

adopted the KD6009A strain amplifier and the KD7901 bridge box as the collection equipment. The strain brick was pre-buried in the concrete model as the sensor to collect dynamic stress signals. In order to prevent the strain brick from being corroded, the strain brick was subject to moisture-proof processing. The test system is shown in Figure 3.

\subsubsection{Loading equipment and model material selection}

Aiming at the propagation and attenuation law of stress waves in fissured rock mass under high confining pressures, this paper carried out an laboratory model experiment, and adopted a deep-rock triaxial dynamic and static load test system to perform the simulation test, as shown in Figure 4, the size of the corresponding model test piece of the equipment was $200 \mathrm{~mm} \times 200 \mathrm{~mm} \times 200 \mathrm{~mm}$, the model test piece was cast by the mold, the surface of the test piece was coated with Vaseline to decrease the end effects of the test piece. The test system is capable of applying three-way uniform loading to the test piece, and applying dynamic loading along the $z$-axis direction. The dynamic load frequency was $50 \mathrm{~Hz}$, and the maximum static load that can be applied was $60 \mathrm{MPa}$. The self-weight of the model was in a smaller magnitude relative to the applied confining pressure, so it can be ignored.

The original rock corresponding to the model test was fissured rock mass under high confining pressure. Through a comprehensive analysis of the mechanical parameters of wall rocks in the deep mining area of a mine in China, according to the relevant theories of the Froude simulation test, it was determined that the test stress scale was 10, and the obtained mechanical parameters of the original rock and the model materials are shown as Table 1 . The model materials were mainly cement mortar, and supplemented by additive materials, wherein the sand was screened by a 24-mesh sieve. The ratio was: cement:sand:water:cement molding agent $=2: 4.7: 1: 0.03$, after the model footnote was completed, sit for 30 days, the measured mechanical

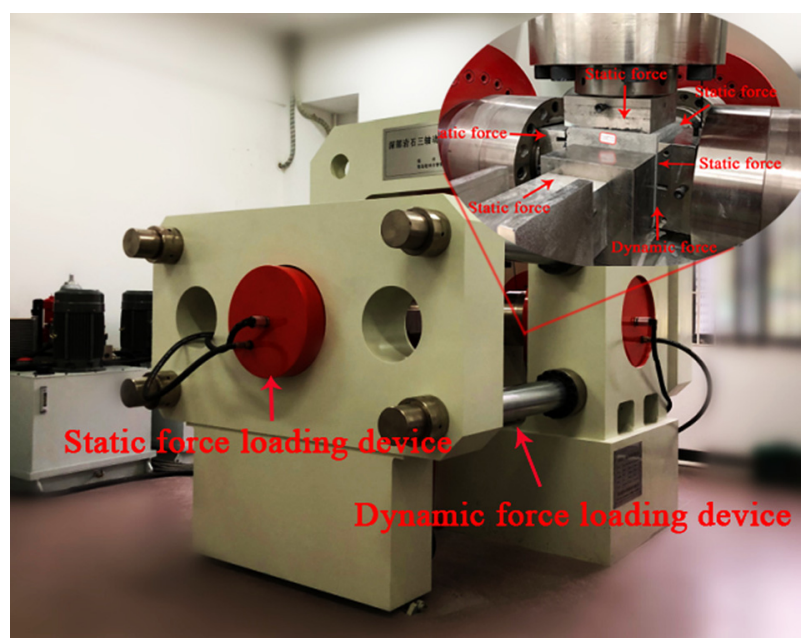

Figure 4: The deep-rock triaxial dynamic and static load test system. 
Table 1: Physical and mechanical parameters of original rock and model similar materials.

\begin{tabular}{lrrrrrrr}
\hline Category & $\begin{array}{r}\text { Compressive } \\
\text { strength }(\mathbf{M P a})\end{array}$ & $\begin{array}{r}\text { Tensile strength } \\
(\mathbf{M P a})\end{array}$ & $\begin{array}{r}\text { Elastic modulus } \\
(\mathbf{G P a})\end{array}$ & $\begin{array}{r}\text { Internal friction } \\
\text { angle }\left(^{\circ}\right)\end{array}$ & $\begin{array}{r}\text { Cohesion } \\
(\mathbf{M P a})\end{array}$ & $\begin{array}{r}\text { Poisson's } \\
\text { ratio }\end{array}$ & $\begin{array}{r}\text { Density } \\
\left(\mathbf{k g ~ m}^{-3}\right)\end{array}$ \\
\hline Original rock & 170.00 & 16.00 & 40.000 & 35.0 & 40.00 & 0.346 & 2800 \\
Model & 17.53 & 1.71 & 1.099 & 30.3 & 2.12 & 0.301 & 2235 \\
\hline
\end{tabular}

parameters are shown in Table 1. The fissures were simulated using mica sheets, via normal loading test, the normal initial stiffness of the fissures was obtained as $k_{\mathrm{ni}}=14 \mathrm{GPa} / \mathrm{m}$. Kulhawy [12] determined the relationship between the shear stiffness and the normal stiffness of the fissure by studying a large number of experimental data, $k_{s}=k_{n} / 2(1-\mu)$, where $\mu$ was the Poisson's ratio of the material, and the calculated shear stiffness of the fissure was $k_{s}=10 \mathrm{GPa} / \mathrm{m}$. In the model test, the confining pressures were: $0 \mathrm{MPa}, 2 \mathrm{MPa}, 4 \mathrm{MPa}$, $6 \mathrm{MPa}, 8 \mathrm{MPa}$, and $10 \mathrm{MPa}$, respectively, and the confining pressures of the corresponding deep rock mass were $0 \mathrm{MPa}, 20 \mathrm{MPa}, 40 \mathrm{MPa}, 60 \mathrm{MPa}, 80 \mathrm{MPa}$, and $100 \mathrm{MPa}$, respectively.

\subsubsection{Experimental program and validation of effectiveness}

The cylindrical stress wave applied to the test piece was generated by the dynamic stress axis of the triaxial dynamic and static load system according to the JWL state equation programming, as shown in Figure 5, wherein the wavelength of the stress wave was $1320 \mathrm{~m}$, which was much larger than the length of the fissure, so the influence of fissure length on the propagation of the stress wave can be ignored; the thickness of the fissures was unified to $5 \mathrm{~mm}$, and the influence of the thickness on the attenuation of the stress wave was ignored as well. Static load was applied to the boundary of the model to simulate the underground confining pressure; the dynamic load was applied by the dynamic axis, and the strain data of each measuring point in the test piece under dynamic load was collected.

The experiment used stress and strain to quantify the change of the stress wave before and after it passing through the fissure. Due to the damping effect of the fissure, the peak of stress and strain mostly appeared in the first wave of the time-history curve, so the ratio of the stress peak value after the fissure and the stress peak value before the fissure was defined as the attenuation factor. In order to avoid the interference of the reflection of the stress wave on the fissure boundary to the collected data, the distance from the measuring point to the fissure should be selected reasonably, so as to eliminate the influence of the reflection wave on the peak value

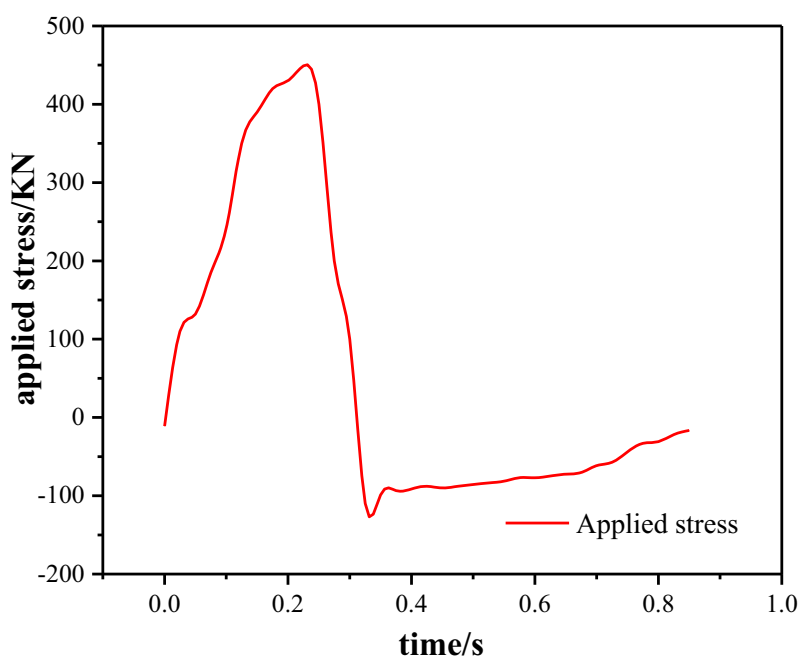

Figure 5: Stress loading path of triaxial compressor in $z$-axis direction. 
of the first wave. The model casting was carried out layer by layer so as to fix the fissure and plant the strain brick; the vibration stirrer was used in the pouring process to ensure that the density of the model was uniform and to reduce the influence of interface delamination on the wave propagation. The effectiveness of stress waves tested by such tests has been verified in previous studies [13].

\subsection{Propagation test of stress wave in fractured rock mass}

Fissures are ubiquitous in underground engineering and are important factors affecting stress propagation. This section summarizes the influence of various fissures on stress propagation attenuation. To study the influence of fissures uniformly distributed in the direction of stress propagation on the stress propagation, one, two, three, four, and five fissures were arranged in the direction of stress propagation to measure the stress peak values before and after the stress wave passing through the fissure group, and the image of stress attenuation was obtained (Figure 6).

The stress wave at the monitoring point 1 is defined as an incident wave, and the stress wave at the monitoring point 2 is defined as a transmitted wave. In order to avoid the interference of reflected waves, the first peak of the stress wave is taken to calculate. The attenuation coefficient obtained by the calculation is organized as shown in Figure 7.

It can be seen from Figure 7 that the attenuation coefficient of the stress wave at the fissures increased with the increase of the confining pressure, which was due to that the increase of the confining pressure had increased the rigidity of the fissure, so that the attenuation coefficient increased. However, fissures will leads to stress decay for stress wave propagate in different materials. As the number of fissures increased, the attenuation coefficient decreased significantly, indicating that the attenuation caused by the increase in the number of fissures was greater than the reduced attenuation caused by the increase in the rigidity of the fissure. In addition, the stress attenuation coefficient decreased by $28.5,35.4,39.2,38.6,35.9,38.5 \%$, respectively with the number of fissures increased from 1 to 5 under the confining pressure of $0,2,4,8$ and $10 \mathrm{MPa}$, this result indicates that the degree of stress attenuation further increases as the confining pressure increases.

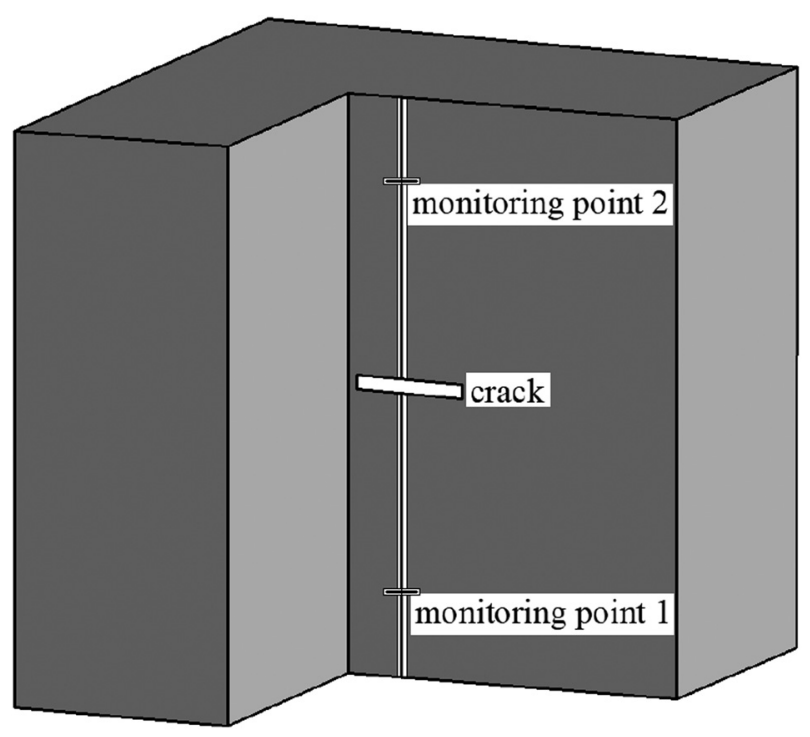

Figure 6: Relative positions of stress-strain sensor and fissure. 


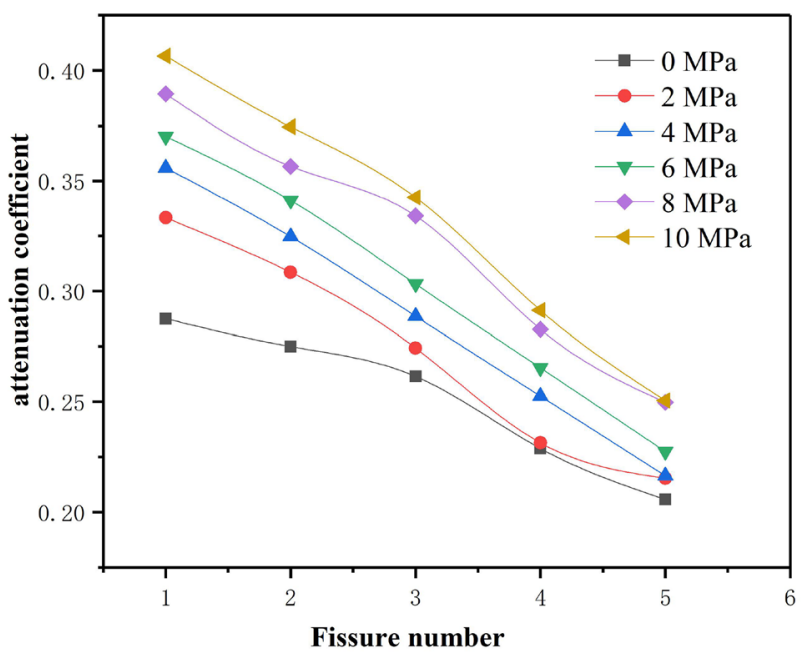

Figure 7: Attenuation law of stress waves in rock mass with different number of fissures.

\section{Numerical simulation analysis}

\subsection{Numerical simulation and results analysis}

Using the ABAQUS/Standard finite element analysis software to establish a numerical model. The geometrical dimensions, crack layout and parameters were consistent with the concrete test model. Limit the displacement of the plane around the model, and the top plane is the non-reflecting boundary.

The peak stress cloud diagram of the impact pressure formed by the bottom dynamic load along the $Z$-axis direction is shown in Figure 8. As can be seen from the figure that the stress tends to decrease along the $Z$-axis direction. The stress wave at the monitoring point 1 is defined as an incident wave, and the stress wave at the monitoring point 2 is defined as a transmitted wave. Calculate the stress attenuation coefficient under various operating conditions, as shown in Figure 9.

It can be seen from Figure 9 that under the effect of the same confining pressure, the stress attenuation coefficient decreases as the number of cracks increases. This is because the deformation occurs as the number of cracks increases and absorbs the energy of the stress wave. On the other hand, when the number of cracks is greater than 1, and the stress wave passes through the cracks, multiple transflections occur between the cracks,
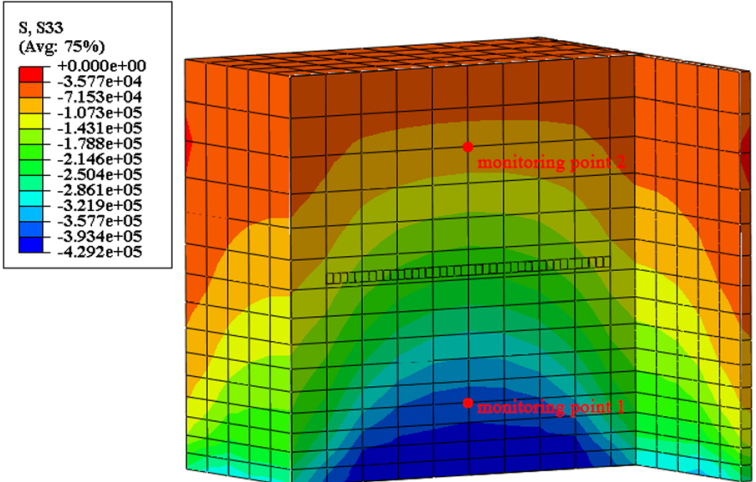

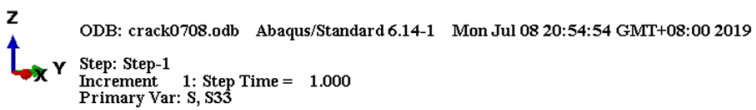

Figure 8: Z-axis stress cloud under $2 \mathrm{MPa}$ confining pressure. 


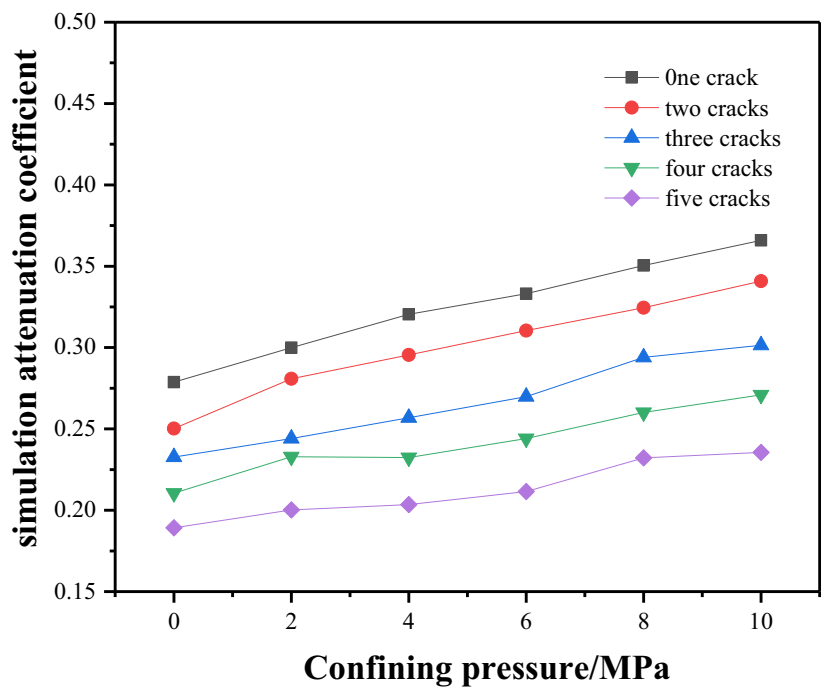

Figure 9: Numerical results of attenuation coefficient with the number of fractures increase.

resulting in a decrease in the attenuation coefficient; Under different confining pressures, the stress attenuation coefficient increases with the increase of confining pressure. This is because the increase of the confining pressure leads to the increase of the nonlinear fracture stiffness, which leads to the increase of the attenuation coefficient.

\subsection{Comparative analysis}

Taking three fissures as an example, in the study, the first wave of the plane stress wave was theoretically analyzed without considering the superposition effect of the stress waves. The stress wave curve of measuring point 2 was taken as the incident stress wave, and the stress wave curve of measuring point 5 was taken as the transmitted stress wave. The working conditions of 0 and $4 \mathrm{MPa}$ confining pressure were selected, the transmitted wave curve of theoretical, experimental and Numerical were drawn as shown in Figure 10:

It can be judged from Figure 10 that the theoretically calculated transmitted stress wave curve was basically consistent with the experimental stress wave curve and numerical simulation stress wave curve, and
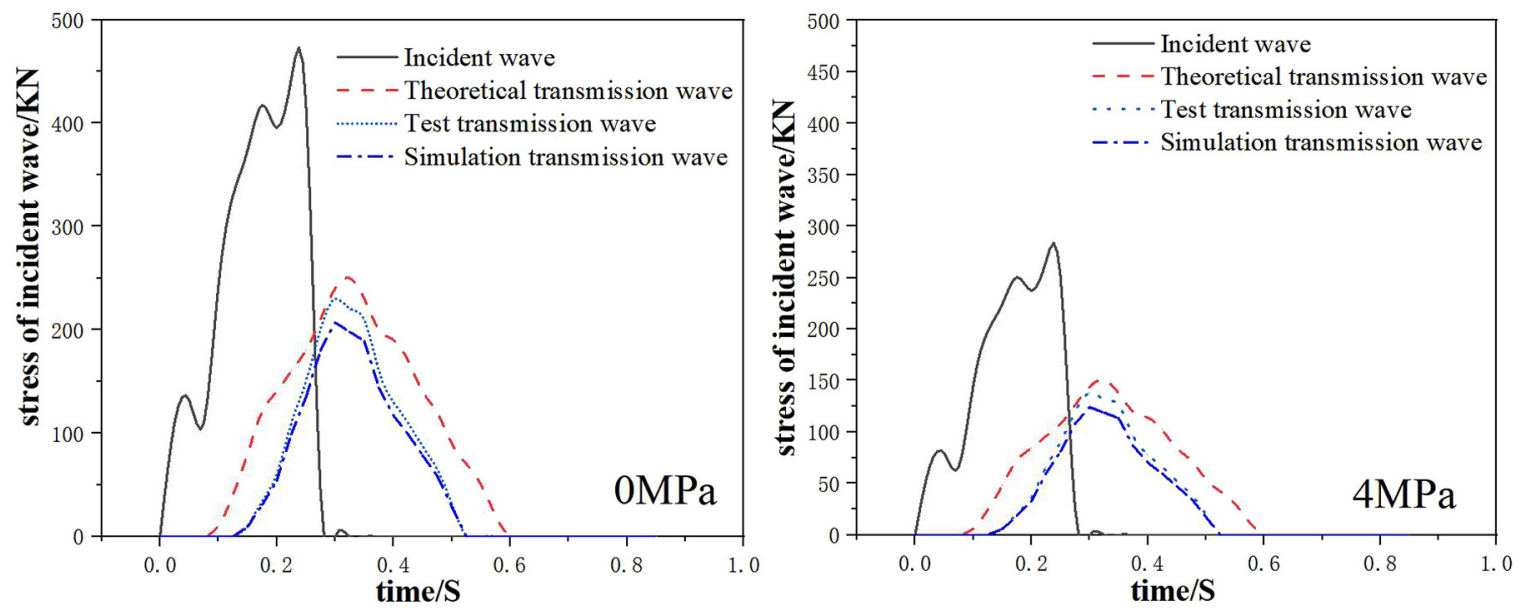

Figure 10: Stress wave curves under $0 \mathrm{MPa}$ and $4 \mathrm{MPa}$ confining pressure. 


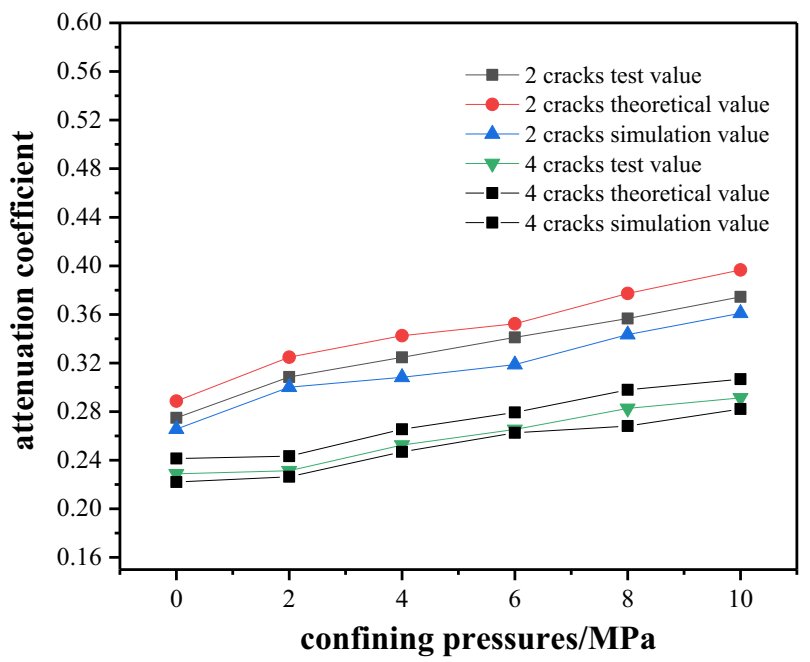

Figure 11: Stress attenuation coefficient in theoretical test and simulation.

there are certain errors in the stress increase and decrease stages. This is because the joint mechanics model adopted in this paper was based on the nonlinear deformation relationship of the joints in the loading stage, while in the unloading and loading stages, there were deviations in the stress deformation path of the joints. The change rate of the deformation of the joints with the decrease of the stress in the unloading stage was greater than that in the loading stage, thus causing the measured decreasing rate of the transmitted wave was faster than the theoretical value in the decrease stage, which is also consistent with the research results of JC Li et al. [14].

\subsection{Summary}

In the experiment and the theoretical calculations of this paper, the influence of the fissures' width and angle was ignored. This paper selected two working conditions: the number of parallel fissures were 2 and 4, and the stress wave was perpendicular to the fissured rock mass. The theoretical value and the measured value of the model were compared and analyzed, and the obtained data is shown as Figure 11.

It can be seen from Figure 11 that the transmission coefficients of cylindrical stress waves in fractured rock masses under different confining pressures are in good agreement 267 with the measured values. Among them, the theoretically analyzed transmission coefficients are slightly larger. As the confining pressure increases, the transmission coefficients increase. This is because the theoretical analysis only considers the radial stress but not the tangential stress, resulting in a larger theoretical value than experiment value. When the number of cracks is small, the theoretical value is basically consistent with the simulation results using the finite software ABQUS. Although for a large number of cracks, the numerical simulation value is slightly smaller than the theoretical value due to the superposition of stress waves, but the trend is basically consistent With the increase of the fracture stiffness, the transmission coefficient shows an increasing trend, and the increasing speed decreases. 


\section{Conclusion}

Based on nonlinear theoretical analysis, model tests and numerical simulation, this paper studied the relationship between the attenuation of stress wave propagation and the number of fissures under different confining pressures, and drew the following conclusions:

(1) Based on the propagation attenuation mechanism of stress waves in fissured rock mass, this paper derived the propagation equations of stress waves passing through multiple fissures, compared the theoretical value with the experimental result and numerical simulation, and found that these three were in good agreement, which had verified the feasibility of theoretically calculating the stress wave attenuation coefficient.

(2) The transmission coefficient of stress wave in fissured rock mass is related to the number of fissures, and it decreases with the increase of the number of fissures. And The degree of stress attenuation further increases as the confining pressure increases.

(3) In this paper, the attenuation law of dynamic stress wave in the region passing through nonlinear fractures is studied by theoretical, laboratory test and numerical simulation. Although the model is simplified to some extent, the regularity of stress wave propagation caused by multiple fractures is effectively and rapidly studied. It has practical significance to study the law of stress wave propagation caused by blasting and the law of field blasting vibration test as well as rock mass stability caused by blasting.

\subsection{Data availability}

The datasets used or analyzed during the current study are available from the corresponding author on reasonable request.

Acknowledgments: This research work was supported by the National Natural Science Foundation of China under Grants 51679093 and 51374112.

Author contribution: All the authors have accepted responsibility for the entire content of this submitted manuscript and approved submission.

Research funding: This research work was supported by the National Natural Science Foundation of China under Grants 51679093 and 51374112.

Conflict of interest statement: The authors declare no conflicts of interest regarding this article.

\section{References}

[1] M. Schoenberg, "Elastic wave behavior across linear slip interfaces,” J. Acoust. Soc. Am., vol. 65, no. 5, pp. 1516-1521, 1980.

[2] J. G. Caj and J. Zhao, "Effects of multiple parallel fractures on apparent attenuation of stress waves in rock masses," Int. J. Rock Mech. Min. Sci., vol. 37, no. 4, pp. 661-682, 2000.

[3] K. Aki and P. G. Richards, Quantitative Seismology, 2nd ed., W. H. Freeman \& Company, 1980, pp. 5-43.

[4] J. C. Li, G. W. Ma, and J. Zhao, "An equivalent viscoelastic model for rock mass with parallel joints," Journal of Geophysical Research: Solid Earth, vol. 115, no. B3, pp. 1923-1941, 2010.

[5] S. C. Bandis, A. C. Lumsden, and N. R. Barton, "Fundamentals of rock joint deformation," Int. J. Rock Mech. Min. Sci. Geomech. Abstr., vol. 20, no. 6, pp. 249-268, 1983.

[6] J. C. Li and G. W. Ma, "Experimental study of stress wave propagation across a filled rock joint," Int. J. Rock Mech. Min. Sci., vol. 46, no. 3, pp. 471-478, 2009.

[7] L. Tingting, L. I. Xinpin, L. I. Haibo, et al., “Numerical study on stress wave propagation across filled joints," Chin. J. Rock Mech. Eng., vol. 35, no. S2, pp. 3552-3560, 2016.

[8] L. J. Pyrak-Nolte, L. R. Myer, and N. G. W. Cook, "Anisotropy inseismic velocities and amplitudes from multiple parallel fractures,” J. Geophys. Res., vol. 95, no. B7, pp. 345-358, 1990.

[9] Y. Jin, S. Boxue, Q. Qian, et al., "Propagation of P-wave in dual nonlinear elastic medium for jointed rock mass," Chin. J. Rock Mech. Eng., vol. 30, no. 12, pp. 2463-2473, 2011. 
[10] J. C. Li, H. B. Li, G. W. Ma, et al., "A time-domain recursive method to analyse transient wave propagation across rock joints," Geophys. J. Int., vol. 188, no. 2, pp. 631-644, 2012.

[11] J. P. Zhao, Experimental Study on Test and Identification of Blast Wave in Adjacent Area to a Borehole in Concrete Model and its Action (Ph.D. Thesis), Changsha, Hunan, Central South University of Technology, 2019.

[12] F. H. Kulhawy, "Stress deformation properties of rock and rock discontinuities," Eng. Geol., vol. 9, no. 4, pp. 327-350, 1975.

[13] X. Lou, R. Luo, and Y. Jin, "Attenuation law of stress waves in cracked rock mass under different confining pressures[J]," Adv. Civ. Eng., 2019, 2019.

[14] J. Li, G. Ma, and X. Huang, "Analysis of wave propagation through a filled rock joint," Rock Mech. Rock Eng., vol. 43, no. 6, pp. 789-798, 2010.

[15] L. Tingting, L. Jianchun, L. Haibo, et al., "Numerical analysis for the effect of stress wave propagation through nonlinear joints [J]," CJRME, vol. 34, no. 05, pp. 953-959, 2015.

[16] C. Shaobo, Z. Junhai, W. Hao, “UDEC simulation on cylindrical wave propagation through jointed rock masses[J]," CJRME, vol. 38, no. S1, pp. 2848-2856, 2019.

[17] C. Shaobo, L. Jianchun, L. Haibo, et al., "Study on stress P-wave propagation across intersecting rock joints with nonlinear deformation[J]," CJRME, vol. 38, no. 06, pp. 1149-1157, 2019.

[18] L. Xinping, D. Qian, H. JunHong, et al., "Research on propagation law of cylindrical stress wave in jointed rock mass under insitu stress[]]," CJRME, vol. 37, no. S1, pp. 3121-3131, 2018.

[19] L. Tingting, L. Xinping, Z. Yun, et al., "Analysis of Seismic waves propagating through an in situ stressed rock mass using a nonlinear model[]]," Am. Soc. Civil Eng., vol. 20, no. 3, 2020. 